\title{
Representaciones sociales y prácticas educativas del profesorado en torno a la educación para la ciudadanía*
}

\author{
Social representations and educational practices of teachers around \\ citizenship education
}

\section{Representações sociais e práticas educacionais de professores sobre educação para cidadania}

\section{**Alejandro Rabuco Hidalgo}

Rabuco Hidalgo, A. (2020). Representaciones sociales y prácticas educati1vas del profesorado en torno a la educación para la ciudadanía. Revista Convergencia Educativa 7, julio, 1-21. DOI: http://doi.org/10.29035/rce.7.2

[Recibido: 11 febrero, 2020 / Aceptado: 26 mayo, 2020]

\section{RESUMEN}

Este estudio presenta una investigación que busca describir y comprender las prácticas educativas y los discursos del profesorado presentes en la enseñanza de la educación para la ciudadanía en Chile. Se plantea la discusión acotada del objeto teórico de la ciudadanía y la política pública chilena relacionada con la enseñanza de formación ciudadana en la escuela. La metodología utilizada es de carácter cualitativa y se centra en el análisis de las entrevistas y la observación de las prácticas pedagógicas de profesores de dos establecimientos públicos y particular subvencionados de la Región de Valparaíso, Chile. Desde donde se levantan categorías matrices ligadas a la escuela, el currículum y la función docente. Los principales resultados se vinculan con la carencia de espacios de diálogos en los establecimientos educativos que contribuyen a la generación de prácticas pedagógicas de formación ciudadanas intuitivas, poco reflexivas, y la ausencia de un correlato formativo de la ciudadanía durante la formación inicial de los docentes.

Palabras clave: formación ciudadana, prácticas pedagógicas, discursos, escuela.

\footnotetext{
*El presente trabajo forma parte de una investigación de tesis elaborado durante los años 2017-2018, para el Programa de Magíster en Educación de la Pontificia Universidad Católica de Valparaíso titulada "Prácticas educativas y discursos docentes en la formación ciudadana: estudio de caso de dos escuelas de la Región de Valparaíso". Trabajo dirigido por el Dr. Félix Angulo Rasco. Cuenta con el financiamiento de la Comisión Nacional de Investigación científica y tecnológica de Chile ( CONICYT). Proyecto N²2170191.
}

**Universidad Autónoma de Barcelona, España. 
Rabuco Hidalgo, A. (2020). Representaciones sociales y prácticas educativas del profesorado en torno a la educación para la ciudadanía.

Revista Convergencia Educativa, 7, julio, 1-16. DOI: http://doi.org/10.29035/rce.7.2

\begin{abstract}
This study presents a research that seeks to describe and understand the educational practices and discourses of teachers present in the teaching of citizenship education in Chile. The limited discussion of the theoretical object of citizenship and Chilean public policy related to the teaching of citizen training in the school is raised. The methodology used is of a qualitative nature and focuses on the analysis of the interviews and the observation of the pedagogical practices of teachers from two public and private subsidized establishments in the Region of Valparaíso, Chile. Matrix categories linked to the school, the curriculum and the teaching function are built. This article brings together the main results, aimed at discussing the lack of dialogue spaces in educational establishments that contribute to the generation of intuitive and non-reflective citizen training pedagogical practices, and the absence of a formative correlation of citizenship during initial training of the teachers.
\end{abstract}

Key words: Citizen education, Pedagogical practices, speeches, school.

\title{
RESUMO
}

Este estudo apresenta uma pesquisa que busca descrever e entender as práticas educacionais e os discursos dos professores presentes no ensino da educação para a cidadania no Chile. Nesse sentido, levanta-se a discussão limitada do objeto teórico da cidadania e das políticas públicas chilenas relacionadas ao ensino da formação do cidadão na escola. A metodologia utilizada é de natureza qualitativa e concentra-se na análise das entrevistas e na observação das práticas pedagógicas dos professores de duas instituições públicas e privadas subsidiadas na região de Valparaíso, Chile. De onde são construídas as categorias matriciais ligadas à escola, o currículo e a função de ensino. Os principais resultados estão relacionados à falta de espaços para diálogos em estabelecimentos de ensino que contribuam para a geração de práticas intuitivas de formação pedagógica, pouco reflexivas, e à ausência de uma correlação formativa da cidadania durante a formação inicial de profesores.

Palavras-chave: Educação cidadã, práticas pedagógicas, discursos, escola.

\section{Introducción}

El presente artículo, es el resultado de un estudio desarrollado en colaboración con el equipo de investigación de la línea dos de educación y ciudadanía del Centro de Investigación para la Educación Inclusiva de la Pontificia Universidad Católica de Valparaíso, vinculado con la educación para la ciudadanía y las practicas docentes. A partir del análisis de los discursos de los docentes y la observación empírica de clases, se buscó analizar las formas en que el profesorado comprende y práctica la enseñanza de la ciudadanía dentro de los contextos escolares.

En el transcurso de este trabajo, surgen categorías relacionadas con las percepciones de los y las docentes en torno a la ciudadanía, vinculadas con las formas en que se comprende el proceso formativo y como se reconoce cada sujeto en la comunidad educativa, vale decir; estar presente en la escuela, sin sentirse parte de la comunidad, participar de proyectos de Formación Ciudadana donde no se reconocen sus creencias y percepciones. Se observa la carencia de vinculación entre los docentes y los proyectos educativos propuestos por las escuelas. Lo anterior, se ve reflejado en el tipo de estrategias didácticas 
Rabuco Hidalgo, A. (2020). Representaciones sociales y prácticas educativas del profesorado en torno a la educación para la ciudadanía.

Revista Convergencia Educativa, 7, julio, 1-16. DOI: http://doi.org/10.29035/rce.7.2

utilizadas en las clases y talleres de Formación Ciudadana, las que se posicionan desde dos vertientes particulares; una ligada a la enseñanza de la ciudadanía política tradicional y otra centrada en una perspectiva social o comunitaria del ser ciudadano, desde donde se busca potenciar la reflexión crítica sobre la realidad social cercana. Esto permite identificar, la construcción de subjetividades que dan cuenta de valoraciones y percepciones diferentes sobre la ciudadanía, las que no son sociabilizadas ni propuestas bajo el trabajo pedagógico colectivo y la aplicación de una estructura curricular transversal. En definitiva, elementos como interdisciplinariedad y transversalidad curricular parecen ser hoy letra muerta, plasmada en la política pública del Plan de Formación Ciudadana chileno.

\section{Antecedentes}

\subsection{La política pública en formación ciudadana desde las prácticas docentes}

En Chile, las autoridades del Ministerio de Educación se han dado a la tarea de vincular la educación de niños y jóvenes, con un proyecto transversal que inste a la comprensión de la ciudadanía como vehículo para la valoración de la democracia y los derechos humanos. Así, desde el plano curricular a través de la formación ciudadana las autoridades de educación han realizado grandes esfuerzos en el diseño y posterior aplicación de estrategias educativas vinculadas con la participación y la democracia en la cultura escolar chilena. Si antes el desafío se dirigía hacia la formación de los estudiantes a través de la educación cívica, ahora, se busca pasar hacia un concepto de formación ciudadana más amplio, inclusivo y profundo, lo que implicaría, el desarrollo de competencias necesarias para la participación social.

Bajo la consigna que dicta de la necesidad de transformar el currículo educativo para el estudio, de la formación ciudadana de forma transversal en los distintos niveles del sistema educativo (Cox, 2003), se ha desarrollado la Ley 20.911 (2016), que insta a los establecimientos escolares a construir proyectos que contengan definiciones teóricas, orientaciones técnicas y pedagógicas para la enseñanza de la ciudadanía en Chile.

Se busca la generación de un diseño curricular que movilice la adquisición por parte de los estudiantes de habilidades, conocimientos y actitudes para fortalecer el sistema democrático y los derechos humanos. Para ello, el Ministerio de Educación recomienda que:

Los establecimientos educacionales reconocidos por el Estado deberán incluir en los niveles de enseñanza parvularia, básica y media un Plan de Formación Ciudadana que integre y complemente las definiciones curriculares nacionales en esta materia, que brinde a los estudiantes la preparación necesaria para asumir una vida responsable en una sociedad libre y de orientación hacia el mejoramiento integral de la persona humana, como fundamento del sistema democrático, la justicia social y el progreso (Ministerio de Educación, 2016, p.7). 
Rabuco Hidalgo, A. (2020). Representaciones sociales y prácticas educativas del profesorado en torno a la educación para la ciudadanía.

Revista Convergencia Educativa, 7, julio, 1-16. DOI: http://doi.org/10.29035/rce.7.2

En base a estos nuevos lineamientos, se espera que los establecimientos educacionales puedan ser transformados en espacios en donde se educa en y para la democracia. Este último punto es fundamental, comprendiendo que Chile ha intentado constantemente superar las graves omisiones heredadas del periodo de dictadura militar y sus políticas represivas, cuyas consecuencias impactan en el diseño de los programas educativos, sobre todo en materias referidas a la valoración y protección de la democracia y los derechos humanos (Muñoz \& Torres, 2014). Es por eso, que los nuevos objetivos y contenidos mantienen una relación estrecha con la participación de los estudiantes en la comunidad educativa, a través de talleres extraprogramáticos y la implementación de distintas iniciativas tendientes a fomentar la organización como los consejos escolares y centros de estudiantes etc.

Uno de los puntos de partida que permite comprender estas nuevas definiciones, es lo que el Ministerio de Educación ha llamado el paso desde la Educación Cívica a la Formación Ciudadana. En dicho análisis, la Educación Cívica tradicionalmente tratada por el currículo escolar se asume como una asignatura en la que predomina la enseñanza centrada en contenidos relacionados con las instituciones del Estado y el futuro ejercicio de los "derecho de ciudadanía plena" lo que en otras palabras significa el ejercicio de sufragio (Ministerio de Educación, 2016, p.20).

La actualización curricular del año 2009 genera una serie de transformaciones en orden a dar forma al diseño de lineamientos que permitieran articular aprendizajes específicos de la economía y la institucionalidad política en la asignatura de Historia y Cs. Sociales para Cuarto año Medio. Lo antes señalado, tiene sustento en las propuestas manifestadas por la Comisión de Formación Ciudadana (2004), organización que vio en los resultados de las pruebas internacionales de CIVED y el ICCS (2010), elementos importantes para determinar que caminos seguir en la búsqueda de fortalecer la educación ciudadana en Chile. En este caso, se propuso generar los incentivos necesarios para fomentar el desarrollo de habilidades y competencias en los estudiantes.

A este respecto, la propuesta curricular del año 2009 en Chile se ajusta a las demandas presentadas por diferentes organismos internaciones relacionadas con la baja participación de los jóvenes en los procesos políticos formales y el fortalecimiento de la democracia. Se plantea que una democracia robusta exige la participación activa de su ciudadanía en los asuntos público (Bolívar, 2007). Si bien, las políticas educativas nacionales centran sus objetivos en una percepción hegemónica de la ciudadanía liberal en el sistema escolar, lo que a nivel internacional se comienza a promover es la idea de una ciudadanía activa, comprometida y critica que tiende a profundizar los pilares fundamentales del sistema democrático, aspectos que, para el caso chileno, se pretende trabajar involucrando a la comunidad educativa en el proceso de formación de ciudadanos para un mundo globalizado. 
Rabuco Hidalgo, A. (2020). Representaciones sociales y prácticas educativas del profesorado en torno a la educación para la ciudadanía.

Revista Convergencia Educativa, 7, julio, 1-16. DOI: http://doi.org/10.29035/rce.7.2

La Formación Ciudadana a diferencia del civismo, representa una concepción más amplia de aquello que implica la ciudadanía, no tan solo en términos de contenidos, habilidades y aptitudes, sino también en términos de derechos y obligaciones al problematizar la sociedad democrática y su sentido de comunidad, diferencias tratadas en el siguiente cuadro:

\section{Cuadro 1}

Objetivos y contenidos educación cívica y formación ciudadana.

\begin{tabular}{|c|c|c|}
\hline & Educación Cívica & Formación Ciudadana \\
\hline \multirow[t]{3}{*}{ Temas } & Institucionalidad política & Institucionalidad política. \\
\hline & & Convivencia y participación en una sociedad democrática. \\
\hline & & $\begin{array}{l}\text { Problemas actuales de la sociedad, tales como distribución de } \\
\text { ingreso, derechos humanos, equidad, medioambiente, etc. }\end{array}$ \\
\hline Foco de aprendizaje & $\begin{array}{l}\text { Orientada a la adquisición } \\
\text { de conocimiento a través } \\
\text { de los contenidos. }\end{array}$ & $\begin{array}{l}\text { Orientada a la adquisición de conocimientos, habilidades y actitudes } \\
\text { que consideren el contexto y los intereses de las y los estudiantes, a la } \\
\text { promoción de prácticas con predominio de relaciones participativas } \\
\text { y democráticas, y a las competencias para resolver conflictos. }\end{array}$ \\
\hline
\end{tabular}

Fuente: Ministerio de Educación Chile, 2016.

De acuerdo por lo descrito en las indicaciones pedagógicas para la enseñanza de la educación para la ciudadanía, se puntualiza en la importancia de innovar en las estrategias que el docente pone en práctica en el aula. Como sostienen Pagès y Santisteban (2004), el termino educación para la ciudadanía incorpora un enfoque vinculado a la preparación para la participación democrática que no existía en el modelo más clásico de la instrucción cívica, dedicado básicamente al conocimiento del funcionamiento institucional. Al hablar de educación para la ciudadanía, se involucran nuevas formas de entender la ciudadanía, la enseñanza y a los mismos estudiantes; obligando al docente y a la escuela a potenciar las experiencias en sociedad, donde el alumnado pueda vivenciar la participación.

La ciudadanía es presentada como un área de formación que involucra a los diversos actores de la comunidad escolar. Por esto, se espera que el docente pueda fortalecer esta concepción a partir de las experiencias de trabajo de los propios estudiantes (Ministerio de Educación, 2016). Se entiende el espacio escolar y el aula como un lugar abierto y reflexivo, en el que se deben potenciar los principios democráticos y los derechos humanos, proponiendo un trabajo didáctico centrado en los estudiantes, teniendo claridad sobre las necesidades, habilidades e intereses de ellos como participantes del proceso formativo, y el docente es presentado como facilitador y mediador de aprendizajes significativos y acordes a la realidad de los estudiantes. 
Rabuco Hidalgo, A. (2020). Representaciones sociales y prácticas educativas del profesorado en torno a la educación para la ciudadanía.

Revista Convergencia Educativa, 7, julio, 1-16. DOI: http://doi.org/10.29035/rce.7.2

En el ámbito de las estrategias educativas, East \& Evans (2010) concluyen que las metodologías didácticas participativas, democráticas e inclusivas, se han transformado en una necesidad profunda e inminente para todo sistema educativo que tenga por objetivo, la promoción del conocimiento y el compromiso ciudadano. Lo antes señalado, pone de manifiesto la necesidad profunda de resignificar la escuela como espacio de construcción de ciudadanía, y el rol del profesorado en esta tarea como agente movilizador de oportunidades para el desarrollo de competencias sociales en los y las estudiantes (Redon, 2010). Frente a este desafío, es relevante puntualizar en la existencia de diversas formas de comprender la enseñanza de la formación ciudadana, las cuales remite a diferentes concepciones o énfasis en torno a lo que debe ser el rol de la institución escolar, así como las concepciones de sujeto, democracia y ciudadanía (Cerda, Egaña, Magendzo, Santa Cruz \& Varas, 2004).

Algunas aproximaciones de indagaciones realizadas en el campo escolar (Cerda et al., 2004, Cox y Castillo, 2015), identificaron categorías concretas vinculadas a las formas en que el profesorado presenta la enseñanza de la Formación ciudadana en las aulas, las que se diferencian en sus contenidos, proyecciones y orientaciones pedagógicas. A continuación se presentan tres categorías propuestas en el trabajo de campo desarrollado por Cerda et al. (2004):

a) Formación para la Ciudadanía política: modelo que sustenta sus principios políticos y filosóficos en las visiones liberales y jurídicas en torno a la ciudadanía, desde donde surge como emblema educativo la tradicional Educación Cívica. Teoría que apunta a la formación de los estudiantes bajo la comprensión y valoración de las instituciones democráticas, lo que Prats (2012) menciona citando a Habermas como "patriotismo constitucional".

b) La Formación para la Ciudadanía Social: es otra de las categorías elaboradas, perspectiva que desarrolla un modelo de ciudadanía a través de la incorporación en su estudio de elementos problematizadores de los procesos sociales. Esta noción, posibilita una lectura más comprensible de la relación entre los derechos del hombre y la ciudadanía, argumentando, que no es posible pensar en los derechos de las personas basándonos exclusivamente en lo constitucional y lo político. La Formación para una Ciudadanía Activa, es otra categoría que comprende metodológicamente la Formación Ciudadana desde el principio de la participación como herramienta para la construcción de la sociedad, ya que la sola entrega de conocimientos en base a derechos civiles y sociales no basta, la ciudadanía y la educación se deben pensar como un medio o modo de convivencia social basado en la democracia (Cerda et al., 2004).

c) La Formación para la ciudadanía desde una postura crítica: concepto que es levantado a la luz de postulados que proponen la transformación de la escuela en función del cambio social. Así, se pretende incluir en el proceso de educación para la ciudadanía la problematización de las relaciones 
Rabuco Hidalgo, A. (2020). Representaciones sociales y prácticas educativas del profesorado en torno a la educación para la ciudadanía.

Revista Convergencia Educativa, 7, julio, 1-16. DOI: http://doi.org/10.29035/rce.7.2

sociales y el ejercicio del poder, buscando a través de este ejercicio, la transformación de los elementos que subyacen al modelo tradicional de educación y pedagogía.

La revisión de las políticas públicas y estudios internacionales referentes a la educación cívica y la formación ciudadana, como las emanadas desde La Comisión de Formación Ciudadana en Chile (2004), el Estudio Educación Cívica CIVED (ICCS, 2010). y la Encuesta Internacional de enseñanza y aprendizaje TALIS (OCDE, 2009). Todos instrumentos y documentos que concuerdan en sus diferentes contextos, en que unos de los principales desafíos son representados por la generación de mecanismos que permitan innovar en la práctica pedagógica, con intención de despertar el interés en los estudiantes sobre el aprendizaje en formación ciudadana, para lo cual, es necesario la integración de métodos más demandantes que el solo traspaso de información y conocimientos (Cox y Castillo, 2015).

\section{Aspectos Metodológicos}

El enfoque propuesto para esta investigación es de carácter cualitativo y responde al paradigma interpretativo de investigación, toda vez que se busca indagar sobre el fenómeno de la Formación Ciudadana, a través de la comprensión de las dinámicas presentes en los contextos y los elementos que subyacen a los discursos de los sujetos. La metodología seleccionada es el estudio de caso, siendo su objetivo metodológico central, la descripción y comprensión de las formas en que es abordada la enseñanza de la formación ciudadana, a partir del análisis de los discursos, las percepciones de los docentes y la observación de las estrategias didácticas utilizadas en el aula.

\subsection{Diseño y criterios de selección}

Asumiendo la importancia del principio de heterogeneidad que permita la inclusión de sujetos diversos en la investigación, se optó por una unidad de estudio compuesta por dos establecimientos educativos; uno de administración municipal y otro particular- subvencionado. Teniendo presente que el objetivo de estudio apunta a describir y comprender las prácticas y discursos que se promueven desde la educación para la ciudadanía, se han realizado un total de 11 observaciones en aula y 10 entrevistas al profesorado, divididas entre ambos tipos de establecimientos educativos. Además, con la intención de ampliar el rango de comprensión sobre las maneras en que es abordada la Formación Ciudadana en el contexto escolar, se registra asistencia en actividades extraprogramáticas organizadas por los diferentes profesores y encargados de ciudadanía. De esta forma, lo describe el cuadro 2 de registro de entrevistas y actividades: 
Rabuco Hidalgo, A. (2020). Representaciones sociales y prácticas educativas del profesorado en torno a la educación para la ciudadanía.

Revista Convergencia Educativa, 7, julio, 1-16. DOI: http://doi.org/10.29035/rce.7.2

Cuadro 2

Registro de entrevistas.

Registro de entrevistas y actividades

\begin{tabular}{|c|c|c|c|c|}
\hline & Nombres & $\begin{array}{l}\text { Cantidad de } \\
\text { entrevistas }\end{array}$ & Cursos & Registro de actividades \\
\hline \multirow{5}{*}{ Establecimientos públicos } & Camila & 1 & $8^{\circ}$ Básico & \multirow{5}{*}{$\begin{array}{l}\text { - Conmemoración semana de la migración. } \\
\text { - Celebración de Fiestas patrias } \\
\text { - Conmemoración año nuevo indígena. } \\
\text { - Salida a terreno, barrio puerto Valparaíso. }\end{array}$} \\
\hline & Nicolás & 1 & $1^{\circ}$ Medio & \\
\hline & Ester & 1 & $8^{\circ}$ Básico & \\
\hline & Francisco & 1 & $8^{\circ}$ Básico & \\
\hline & Pedro & 1 & $1^{\circ}$ Medio & \\
\hline \multirow{5}{*}{$\begin{array}{c}\text { Establecimientos } \\
\text { particular-subvencionados }\end{array}$} & Esteban & 1 & $1^{\circ}$ Medio & - Celebración semana del medioambiente. \\
\hline & Carolina & 1 & $1^{\circ}$ Medio & \multirow{3}{*}{$\begin{array}{l}\text { - Actividad día del estudiante. } \\
\text { - Salida a terreno Congreso Nacional. } \\
\text { - Salida a terreno Museo de Historia Natural. }\end{array}$} \\
\hline & Daniel & 1 & $8^{\circ}$ Básico & \\
\hline & Natalia & 1 & $8^{\circ}$ Básico & \\
\hline & Javiera & 1 & $1^{\circ}$ Medio & - Elecciones Centro de Estudiantes \\
\hline & Total & 10 & 10 & Total $=6$ \\
\hline
\end{tabular}

Para este trabajo de campo, se han elegido profesores que se encuentran ejerciendo docencia en talleres y asignaturas de formación ciudadana, ligados a disciplinas del área de humanidades, en este caso, profesores de Historia y Cs. Sociales, Arte y tecnología, Filosofía y Educación General básica, principalmente por la cercanía que estos docentes presentan con el mundo de la Formación Ciudadana y los contenidos que en cada una de sus asignaturas se abordan, las que se relacionan directamente con el estudio del hombre en sociedad, sus procesos y complejidades subyacentes.

Por último, para el proceso de observación en aula y las entrevistas realizadas al profesorado participante se entregaron consentimientos informados, en los que se describe la confidencialidad de la información entregada y registrada en las bitácoras, así como el uso exclusivo de los resultados en este estudio. Dicho consentimiento informado fue entregado a los participantes y directivos de los establecimientos involucrados en la investigación para su posterior firma en dos ejemplares 
Rabuco Hidalgo, A. (2020). Representaciones sociales y prácticas educativas del profesorado en torno a la educación para la ciudadanía.

Revista Convergencia Educativa, 7, julio, 1-16. DOI: http://doi.org/10.29035/rce.7.2

\section{Resultados y discusión}

La información recogida en su totalidad forma parte de un apartado extenso, definido por reflexiones sustentadas en diversas categorías. Sin embargo, Debido a las características estructurales que limitan la extensión de este artículo, sólo se comenta el análisis relacionado con la categoría estrategias didácticas.

Durante el proceso de investigación de campo, se evidencia el surgimiento de concepciones políticas y sociales que inciden en los procesos de enseñanza y aprendizaje de las Formación Ciudadana. La información empírica disponible ha permitido el establecimiento de tres desafíos presentes en la enseñanza de la Formación Ciudadana, los cuales más que características comunes, permiten generar una aproximación a la enseñanza de la ciudadanía desde dos contextos educativos particulares, la educación pública y particular subvencionada.

A continuación se presenta el análisis comparativo de los tres desafíos pedagógicos presentes en el diseño y práctica de la educación para la ciudadanía de los dos establecimientos abordados, a partir de los discursos de los docentes y el levantamiento de categorías analíticas propias del proceso de observación en aula

- Enseñanza de la Formación Ciudadana desde la improvisación y/o intuición pedagógica

Las prácticas utilizadas por los docentes en la formación ciudadana, evidencian la necesidad de promover mayores instancias de diálogos y discusiones desde donde se promuevan experiencias didácticas para la formación ciudadana, que permitan retroalimentar a los profesores y de esta forma poner en prácticas en sus respectivas comunidades educativas. De igual forma, a través de las entrevistas se da cuenta de carencias de capacitación en ámbitos ligados a estrategias didácticas y el conocimiento de las políticas públicas sobre formación ciudadana, situación que los profesores perciben como una problemática relevante, mencionando que uno de los desafíos que más cuesta asumir en los proyectos es la necesaria innovación, ya que la mayoría de los profesores no cuenta con experiencias previas sobre enseñanza y práctica de la Formación Ciudadana tal y cual lo plantean las autoridades en las Orientaciones Curriculares.

XX: En la Universidad que abordáramos esta temática no, así contenidos no. Ósea lo que se da transversalmente que es relacionarte con compañeros ver temas políticos, situaciones que ocurren dentro de la universidad y que hay que resolver que se yo, pero así como algo puntual un 
Rabuco Hidalgo, A. (2020). Representaciones sociales y prácticas educativas del profesorado en torno a la educación para la ciudadanía.

Revista Convergencia Educativa, 7, julio, 1-16. D0I: http://doi.org/10.29035/rce.7.2

concepto de democracia, ciudadanía o derechos humanos que hayamos visto no.... ${ }^{1}$ (Entrevista profesora Camila).

Lo anterior, desde el campo de investigación emerge a partir de las pocas experiencias que los docentes han tenido con la educación para la ciudadanía durante su formación inicial. Necesariamente la vinculación de la pedagogía con la formación ciudadana debe ser continua durante el proceso formativo en los futuros profesores. Toda vez, que la mayor parte de los profesores entrevistados reconoció no haber cursado cátedras o seminarios sobre esta temática en su etapa universitaria, lo que, a juicio de los mismos profesores, repercute en que se trabaje la ciudadanía "casi de forma intuitiva", por lo general no centradas en el conocimiento pedagógico, sino que en experiencias de formación escolar. El desarrollo de Planes de Formación Ciudadana donde la evaluación de las practicas docentes no se encuentran presente, ni el dialogo y acuerdos con la comunidad educativa, repercute en las posibilidades de innovar sobre las estrategias educativas.

XX: Como no nos entendemos, en el fondo somos un barco que no tiene para dónde ir, navegamos porque sabemos que tenemos que navegar, vamos a hacer votación de centro de estudiantes porque tenemos que hacerla, pero nos quedamos en seguir las normas establecidas y no actuamos sobre el fondo de lo que es ser ciudadano...2 (Entrevista profesor Nicolás).

- Formación para la Ciudadanía política v/s educación para la ciudadanía desde el territorio y el reconocimiento social

Los resultados del análisis en la observación de clases y las entrevistas muestran formas de presentar la formación ciudadana desde dos vertientes distintas, una ligada al reconocimiento y la participación en la comunidad y la otra enquistada en la tradicional Educación cívica. En ambas concepciones se aprecian culturas escolares opuestas; por un lado una escuela municipal en donde predomina la normalización y escolarización de las conductas, a través de la construcción de normas de convivencia escolar centradas en la autoridad y donde además no se potencia la participación de las estudiantes ni se promueven espacios democráticos de sociabilización. Por otro lado, un colegio particular- subvencionado con una cultura escolar orientada a la generación de diálogos y participación entre docentes y estudiantes como principios de fortalecimiento de la comunidad educativa, espacio en el que los estudiantes y apoderados han logrado desarrollar espacios democráticos para la toma de decisiones.

\footnotetext{
${ }^{1}$ Referencias codificadas [ Cobertura $2.11 \%$ ].

${ }^{2}$ Referencia codificada [ Cobertura 2,28\%].
} 
Rabuco Hidalgo, A. (2020). Representaciones sociales y prácticas educativas del profesorado en torno a la educación para la ciudadanía.

Revista Convergencia Educativa, 7, julio, 1-16. DOI: http://doi.org/10.29035/rce.7.2

XX: Si apelamos exclusivamente al concepto de ciudadanía como al que tiene derecho a participar y vincularse en el mundo adulto (política), es como un concepto más bien técnico que trabajamos en clases para que los chiquillos puedan comprender la importancia por ejemplo de ir a votar.... ${ }^{3}$ (Entrevista profesor Nicolás).

Uno de los argumentos que asoman con mayor fuerza es la necesidad de superar el paradigma tradicional de la educación cívica, que se encuentra presente en la enseñanza de la ciudadanía. En el caso de los procesos pedagógicos observados en el establecimiento público, la Formación Ciudadana es presentada con fuertes énfasis en la transmisión de conocimiento de política institucional a través de la memorización de contenidos vinculados con la historia de Chile y el mundo. Algunos de estos elementos son presentados en la Figura 1 de las categorías emergentes en el modelo de Educación Cívica.

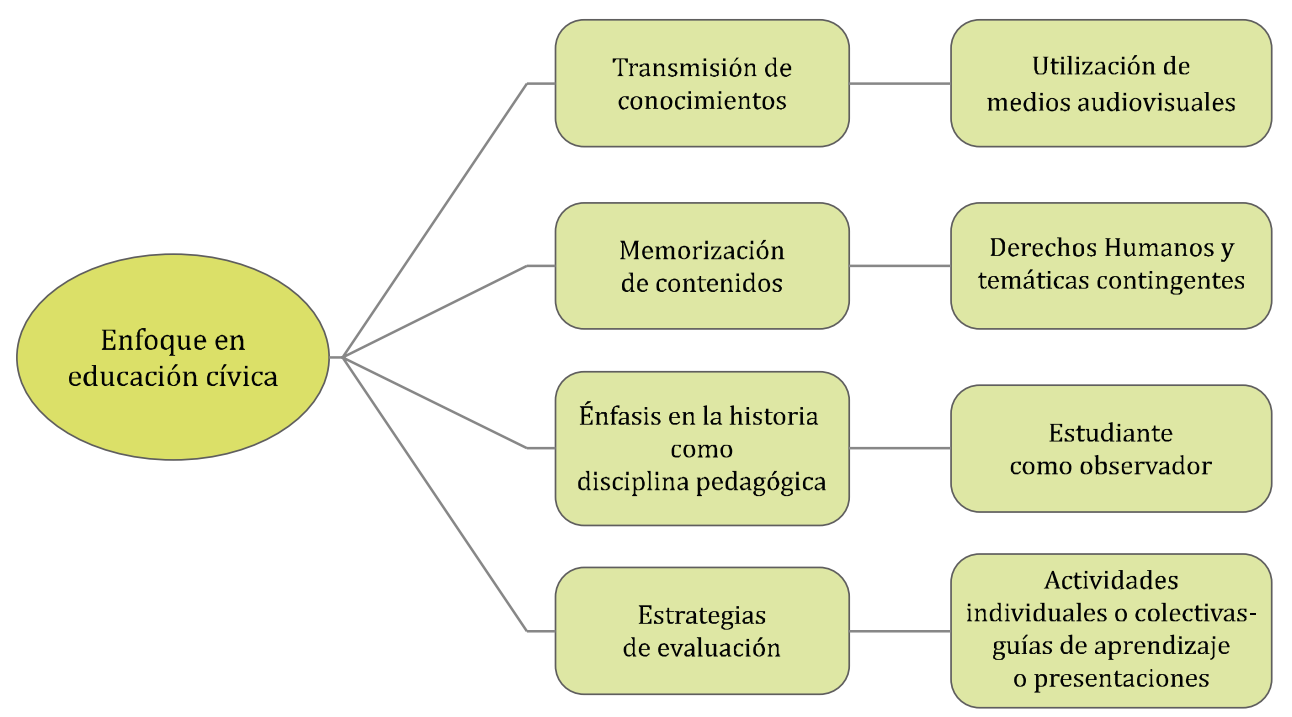

Figura 1. Categoría emergentes modelo de educación cívica.

Participación, patrimonio y espacio público, son algunos de los conceptos que se repiten en las definiciones de los profesores involucrados en este estudio. Se menciona la participación como un elemento central del proceso de formación ciudadana, la que en términos propios los establecimientos hoy han desarrollado a partir de la inclusión de actividades extracurriculares o de beneficencia. Sobre el patrimonio,

\footnotetext{
${ }^{3}$ Referencia codificada [ Cobertura 1,99\%].
} 
Rabuco Hidalgo, A. (2020). Representaciones sociales y prácticas educativas del profesorado en torno a la educación para la ciudadanía.

Revista Convergencia Educativa, 7, julio, 1-16. DOI: http://doi.org/10.29035/rce.7.2

la investigación a través del proceso de observación permite identificar, un énfasis en la importancia de que los estudiantes experimenten la ciudadanía analizando críticamente su contexto social más cercano, más que normas a seguir, la ciudadanía implica la construcción de una comunidad, fruto de la construcción colectiva, desde donde salen los estudiantes para intervenir en el espacio público.

XX: Yo hago intervenciones urbanas con los chiquillos, salimos a la calle, tienen que mirar su recorrido diario para poder analizar desde su percepción los lugares que a ellos les llaman la atención o los lugares que recorren siempre y tratar de dejar una huella dentro de su intervención, porque muchas veces nosotros avanzamos por un lugar no nos damos cuenta, o por las cosas que ocurren, entonces desde ese punto de vista existe la capacidad de ver, pero yo creo que podría mejorarse mucho, porque yo creo que podría ser mucho más intencionado si yo supiera un poco más sobre ciudadanía y como enseñarla... ${ }^{4}$ (Entrevista profesora Ester).

Por otra parte, el análisis de los discursos y observaciones de clases del profesorado del establecimiento particular subvencionado permiten identificar metodologías de enseñanza vinculadas con el estudio de problemáticas sociales relevantes, a partir, del estudio del patrimonio cultural y la motivación por la participación social. La Figura 2 resume los elementos principales de su propuesta didáctica.

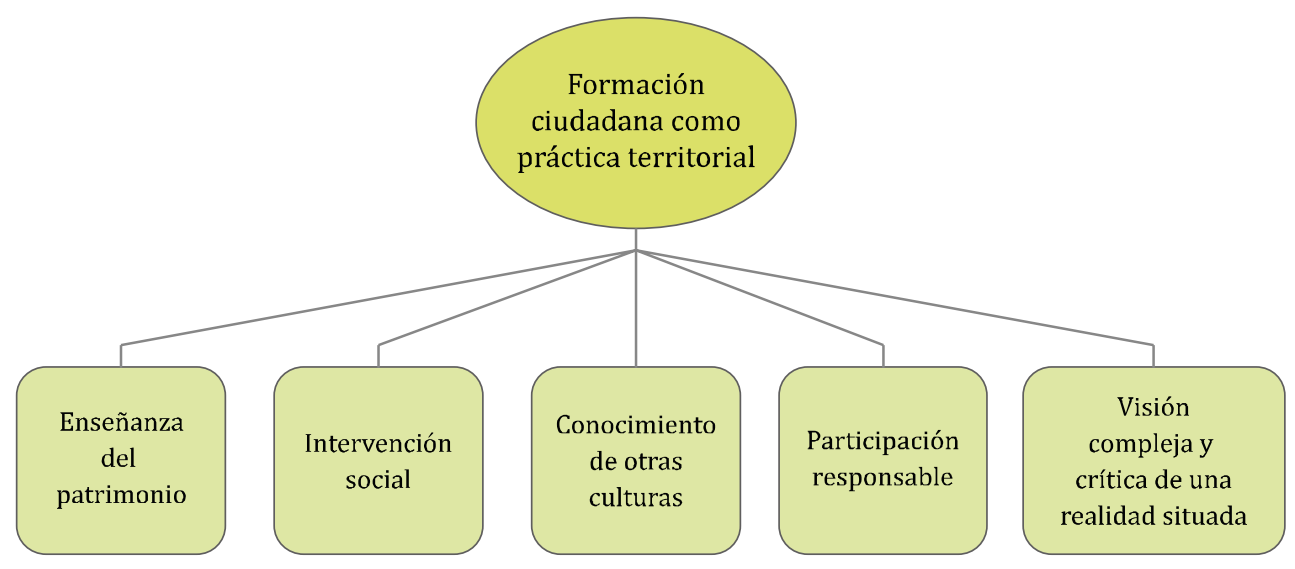

Figura 2. Metodologías de Enseñanza de la Formación Ciudadana ligadas al territorio.

- Interdisciplinariedad y transversalidad en la Formación Ciudadana asignatura pendiente

${ }^{4}$ Referencia codificada [Cobertura $\left.0.94 \%\right]$. 
Rabuco Hidalgo, A. (2020). Representaciones sociales y prácticas educativas del profesorado en torno a la educación para la ciudadanía.

Revista Convergencia Educativa, 7, julio, 1-16. DOI: http://doi.org/10.29035/rce.7.2

La falta de profundización en los conocimientos de los profesores sobre Formación Ciudadana, corre el riesgo de acentuar la fractura existente entre discurso y práctica pedagógica visibilizada en la formación ciudadana. Mientras los docentes, exteriorizan un discurso y racionalidades que posicionan a la ciudadanía desde la transformación e intervención en los espacios públicos, desde la participación y organización activa de los estudiantes, en el aula, las dinámicas observadas reflejan otra realidad, una práctica docente limitada por las relaciones jerarquizadas entre docentes y estudiantes, basadas en el respeto a la autoridad, y en donde lo que predomina, es el énfasis por la transmisión de conocimientos sobre la base de una ciudadanía hegemonizada por el correlato jurídico- político, en donde la reflexión y el análisis pasan a segundo plano.

XX: Sin embargo causa un poco de ruido la perspectiva que se le quiere dar desde el ministerio, yo creo que sin mucho análisis y desde donde nace la Ley 20.911 (2016) tiene que ver con transformar la participación en un ámbito de lecciones, no ahondando por ejemplo en la construcción de redes comunitarias o en el trabajo con los demás colegas que en este colegio al menos es súper complicado, por la burocracia de tener que estar llenado papeles o porque a los colegas no les gusta que uno intervenga sus espacios... ${ }^{5}$ (Entrevista profesor Francisco).

La construcción de proyectos motivados por el cumplimiento de la normativa legal es evidenciada por los mismos directivos y docentes, lo que quiere decir que no se trabaja en la formación ciudadana buscando promover verdaderamente principios democráticos, sino más bien, cumplir con el imperativo legal del Ministerio de Educación. Lo anterior, proyecta, que no existan instancias de sociabilización ni reflexión entre los docentes que les permitan discutir en conjunto los objetivos y motivaciones de la formación ciudadana, situación que provoca desinterés sobre todo en algunos docentes, al mismo tiempo que sitúa a la transversalidad e interdisciplinariedad más como un discurso que una práctica educativa.

la mejora de los proyectos de Formación Ciudadana se debe orientar desde la necesaria sistematización de las prácticas educativas, lo que implica la toma de posición consiente sobre el tipo de prácticas que se desarrollan en el aula, la construcción colectiva de los proyectos de ciudadanía y la constante retroalimentación de las prácticas educativas predominantes al interior de los establecimientos educativos, con el objetivo de identificar y generar cambios frente a dinámicas antidemocráticas establecidas en las comunidades escolares. En esta tarea, es imprescindible la universalización de la Formación Ciudadana como una posibilidad real de transformación social a través de la educación.

${ }^{5}$ Referencia codificada [ Cobertura 1,09\%]. 
Rabuco Hidalgo, A. (2020). Representaciones sociales y prácticas educativas del profesorado en torno a la educación para la ciudadanía.

Revista Convergencia Educativa, 7, julio, 1-16. DOI: http://doi.org/10.29035/rce.7.2

\section{Conclusiones}

Desde el análisis de las percepciones y prácticas del profesorado, emergen diferentes racionalidades, vinculadas con la convivencia y el respeto con el otro, elementos que se relacionan con la necesidad de potenciar normas sociales que permitan el desarrollo de la resolución pacífica de los conflictos.

En la totalidad de las entrevistas analizadas, la convivencia escolar surge como un área de conflicto el cual los profesores ven urgencia en sensibilizar a través de la Formación Ciudadana. Desde esta apreciación, la enseñanza de la ciudadanía se transforma en un eje articulador de los objetivos educativos que hoy se espera desarrolle la escuela como espacio de socialización, de valores y principios normativos que permitan potenciar mecanismos de control frente a acciones de violencia o desorden.

Durante el proceso de observación en aula se evidenció la vinculación constante entre ciudadanía y comunidad, conceptos que a pesar de encontrarse interiorizados en gran medida fueron trabajados por carriles diferentes en las asignaturas o talleres. La comunidad es comprendida desde los parámetros de la caridad, del compañerismo y desde el hecho propio de ser estudiantes que comparten un espacio en común. Cuando se trata de abordar la comunidad desde la Formación Ciudadana, la dimensión política se aborda con poca profundidad privilegiando la revisión de contenidos históricos y cívicos, desde donde se pretende formar al "buen ciudadano", quien más allá de problematizar su realidad social, sabe comportarse frente a los demás.

Al mismo tiempo, los profesores con los que se trabajó en esta investigación reconocen no tener mayor conocimiento sobre investigaciones y literatura especializada en el estudio de la ciudadanía y la educación. Mismos resultados surgen cuando se analiza la experiencia de los docentes en su etapa de formación profesional, lo que permite identificar que la Formación Ciudadana en la actualidad sigue siendo una asignatura pendiente en la formación pedagógica de los y las docentes, incluso en áreas que comúnmente han trabajan cotidianamente temáticas referentes los derechos humanos, fenómenos sociales, identidad y culturas, como puede ser las disciplinas ligadas a las humanidades, desde donde los docentes evidencian que existe un vínculo precario entre la formación disciplinar y la educación para la ciudadanía.

La lógica neoliberal con la que es abordada la ciudadanía pone su acento en el sujeto como eje central de la función educativa, desde un paradigma instrumental de la educación para lo cual los y las estudiantes forman parte de un complejo entramado sobre quienes recae la obligación de responsabilizarse cívicamente a través del voto por el devenir de la democracia chilena. Por otra parte, los discursos del profesorado entrevistado dan cuenta de la construcción de un esquema curricular distinto al oficial. En él, la escuela chilena se subsume bajo un sistema educativo capitalista empresarial extremo que pasa por sobre los 
Rabuco Hidalgo, A. (2020). Representaciones sociales y prácticas educativas del profesorado en torno a la educación para la ciudadanía.

Revista Convergencia Educativa, 7, julio, 1-16. DOI: http://doi.org/10.29035/rce.7.2

derechos sociales del profesorado y los mismos estudiantes, provocando graves tensiones que impiden concretar las ideas republicanas sobre las que debería sustentarse una ciudadanía democrática.

Finalmente, los establecimientos sumidos en una lógica de escolarización terminan por situar a los docentes al interior en un espacio panóptico con dinámicas autoritarias, que les despojan de su autonomía profesional para abordar temas sensibles relacionados con la ciudadanía, la democracia y los derechos humanos tal y como exige la ley. Sumado esto, en la mayoría de los casos las trayectorias formativas del profesorado no contemplan capacitación pedagógica en áreas vinculadas con lo político y la ciudadanía.

\section{REFERENCIAS BIBLIOGRÁFICAS}

Bolívar, A. (2007). Escuela y formación para la ciudadanía. Bordon, Revista de pedagogía, 59(2), 353-374. Recuperado de https://recyt.fecyt.es/index.php/BORDON/article/view/36530

Cerda, A., Egaña, L., Magendzo, A., Santa Cruz, E., \& Varas, R. (2004). El complejo camino de la formación ciudadana. Santiago: Lom.

Comisión Formación Ciudadana (2004). Informe Comisión Formación Ciudadana. Recuperado de https://es.slideshare.net/annyhen/informe-formacin-ciudadana-en-chile

Cox, C., \& Castillo, J. (2015). Aprendizaje de la ciudadanía: contextos, experiencias y resultados. Santiago: Ediciones Universidad Católica de Chile.

Cox, C. (2003). El nuevo currículo del sistema escolar. Documento de Trabajo, Recuperado de https://tendenciascurriculares.files.wordpress.com/2013/04/el_nuevo_curriculum_del_sistema_escolar_pdf

East, V., \& Evans, L. (2010). Guía práctica de necesidades educativas especiales. Madrid: Morata.

ICCS (2010). Estudio Internacional de Educación Cívica y Formación Ciudadana. Recuperado de https://ceritoycruz.files.wordpress.com/2013/10/17-estudio-internacional-de-educacic3b3n-cc3advica-yformacic3b3n-ciudadana.pdf

Ley 20.911 (2016). Crea el plan de Formación Ciudadana para los establecimientos educacionales reconocidos por el Estado. Diario Oficial de la República de Chile. Santiago, Chile.

Ministerio de Educación Chile (2016). Orientaciones curriculares para el desarrollo del plan de Formación Ciudadana. Recuperado de https://bibliotecadigital.mineduc.cl/handle/20.500.12365/467

Muñoz, C., \& Torres, B. (2014). La formación ciudadana en la escuela: problemas y desafíos. EDUCARE, 18(2), 233-245. doi: http://dx.doi.org/10.15359/ree.18-2.12 
Rabuco Hidalgo, A. (2020). Representaciones sociales y prácticas educativas del profesorado en torno a la educación para la ciudadanía.

Revista Convergencia Educativa, 7, julio, 1-16. DOI: http://doi.org/10.29035/rce.7.2

OCDE. (2009). Informe TALIS. La creación de entornos efi caces de enseñanza y aprendizaje - Síntesis de los primeros resultados. Recuperado de https://www.oecd.org/centrodemexico/medios/43058438.pdf

Pagès, J., \& Santisteban, A. (2004). La enseñanza y el aprendizaje del tiempo historico en la educaciòn primaria. Cadernos Cedes, 30(82), 281-309. Recuperado de https://www.scielo.br/pdf/ccedes/v30n82/02.pdf

Prats, E. (2012). ¿Educación cívica o educación para la ciudadanía? Lo que acontece en Europa. En JC. González (Cord.), Identidades culturales y educación en la sociedad mundial. España: Universidad de Huelva

Redon, S. (2010). La escuela como espacio de ciudadanía. Revista Estudios Pedagógicos, 36(2), 213-239. DOI: http://dx.doi.org/10.4067/S0718-07052010000200013

\section{Datos de correspondencia}

Alejandro Rabuco Hidalgo

Profesor de Historia y Ciencias Sociales

Máster en Educación Pontificia Universidad Católica de Valaparaíso

Doctorando en Educación Universidad Autónoma de Barcelona

ORCID: https://orcid.org/0000-0001-6678-9778

Móvil: +56975216990

Email: arturo.rabuco@gmail.com 